\title{
Determinación del momento óptimo de saca de reproductores en cuyes criollos (Cavia porcellus)
}

\author{
Determination of the optimal moment for the culling of breeding native \\ guinea pigs (Cavia porcellus)
}

\author{
Diana Quispe $^{1,2,3}$, Roy Sarmiento ${ }^{1}$, Darwin Huamán ${ }^{1}$, Juan Huayhua ${ }^{1}$, Jhon Tapasco ${ }^{1}$
}

\section{Resumen}

En el Banco de Germoplasma de cuyes nativos de la EEA Chumbibamba del Instituto Nacional de Innovación Agraria (Perú) se determinó el momento óptimo técnico y económico para la saca única de reproductores. Se utilizaron 320 cuyes criollos y se aplicó la metodología Fisher - Hotelling, se planteó el modelo bioeconómico de saca única de reproductores a precio constante bajo cuatro escenarios de tasas de rentabilidad. Los resultados del modelo biológico señalan que la vida productiva óptima en hembras ocurre a los 414 días de edad y en los machos a 579 días. Los valores óptimos del modelo bioeconómico para los cuyes por escenarios de rentabilidad señalan que a una tasa de descuento del $9 \%$ el periodo óptimo de saca sería a 502 días; mientras que a la tasa de descuento del $30 \%$ se reduce a 494 días.

Palabras clave: animales menores, cuy nativo, economía familiar, modelo bioeconómico, peso vivo

\footnotetext{
${ }^{1}$ Estación Experimental Agraria Chumbibamba, Dirección de Desarrollo Tecnológico Agrario, Instituto Nacional de Innovación Agraria (INIA), Andahuaylas, Apurímac, Perú

${ }^{2}$ Facultad de Ingeniería, Universidad Nacional Micaela Bastidas de Apurímac (UNAMBA), Abancay, Apurímac, Perú

${ }^{3}$ E-mail: diana.quispe.roque@gmail.com
}

Recibido: 4 de marzo de 2021

Aceptado para publicación: 25 de septiembre de 2021

Publicado: 27 de octubre de 2021

CLos autores. Este artículo es publicado por la Rev Inv Vet Perú de la Facultad de Medicina Veterinaria, Universidad Nacional Mayor de San Marcos. Este es un artículo de acceso abierto, distribuido bajo los términos de la licencia Creative Commons Atribución 4.0 Internacional (CC BY 4.0) [https:// creativecommons.org/licenses/by/4.0/deed.es] que permite el uso, distribución y reproducción en cualquier medio, siempre que la obra original sea debidamente citada de su fuente original 
In the Germplasm Bank of native guinea pigs of the EEA Chumbibamba of the National Institute of Agrarian Innovation (Peru) the optimal technical and economic moment for the single culling of breeding animals was determined. In total, 320 Creole guinea pigs were used and the Fisher-Hotelling methodology was applied, and the bioeconomic model of single harvest of breeding animals at constant price was proposed under four profitability rate scenarios. The results of the biological model indicate that the optimal productive life in females occurs at 414 days of age and in males at 579 days. The optimal values of the bioeconomic model for guinea pigs by profitability scenarios indicate that at a discount rate of $9 \%$, the optimal harvest period would be 502 days; while at the discount rate of $30 \%$ it is reduced to 494 days.

Key words: small animals, indigenous guinea pig, family economy, bioeconomic model, live weight

\section{INTRODUCCIÓN}

El cuy (Cavia porcellus), pequeño mamífero originario de los Andes central y septentrional de América del Sur produce carne de alto valor nutricional $(20.3 \%$ de proteína) y contribuye a la seguridad alimentaria de las familias rurales y periurbanas de escasos ingresos. El Perú posee una población de cerca de 13 millones de cuyes, estando el $72 \%$ en la sierra hasta los $4500 \mathrm{~m}$ de altitud, formando parte de la economía doméstica y cumpliendo funciones agroecológicas y sociales (Pomboza-Tamaquiza et al., 2016). La mayor población se encuentra en las regiones de Cajamarca (19\%), Cusco (13.5\%), Ancash (12.9\%), Apurímac (8\%) y Junín (7.6\%) (INEI, 2012).

Entre las ventajas comparativas del cuy están el ciclo reproductivo corto, las altas tasas reproductivas, celo en el post partum y poliestricidad, además de ser multíparas. Estos animales son herbívoros, requieren poco espacio en su crianza y no compiten con los animales monogástricos (Aliaga et al., 2009). La crianza del cuy demanda bajos niveles de inversión, pero ofrece un flujo continuo de capital (Sandoval et al., 2013).
El cuy tiene múltiples usos, siendo el más importante la producción de carne, sea como actividad complementaria a la agricultura o como un activo de reserva para las familias (Sánchez y Martínez, 2014), permitiendo la diversificación del ingreso en las unidades familiares (FAO, 2012; Avilés et al., 2014).

En el Perú se encuentran dos genotipos de cuyes: el criollo denominado nativo es pequeño, rústico, poco exigente en calidad de alimento y se desarrolla bien en condiciones ambientales adversas; y el mejorado es precoz por efecto de la selección, muestra mayor productividad (Aliaga et al., 2009). Los cuyes mejorados duplican sus pesos en menor tiempo, tienen eficiente conversión alimenticia y presentan una buena conformación corporal (Chauca, 1994).

El Instituto Nacional de Investigación Agraria (INIA) es pionero es trabajos de investigación para la mejora productiva del cuy (INIA, 2018), a través de las Estaciones Experimentales Agrarias (EEA) como La Molina (Lima), Baños del Inca (Cajamarca), Santa Ana (Junín), Canaán (Ayacucho), Chumbibamba (Apurímac), Andenes (Cusco) e Illpa (Puno). Existen ecotipos locales en las estaciones experimentales del INIA que 
permiten revalorar y fomentar la mejora genética, así como para la transferencia tecnológica (Chauca, 2007). El INIA ha logrado avances en el manejo y mejora genética de razas y líneas de alta productividad como la raza Perú, caracterizada por su precocidad, la raza Andina por su prolificidad, la raza Inti por ser tanto precoz como prolífica (Chauca, 2020).

La modelización bioeconómica en los sistemas de producción animal permite optimizar el uso racional de los recursos zoogenéticos. Por ejemplo, los modelos de extracción y/o uso sostenible de los recursos naturales como madera y pesca permiten determinar los valores óptimos a nivel técnico y económico para la extracción del recurso natural (Jørgensen y Bendoricchio, 2001). En la práctica, diversos modelos matemáticos han sido utilizados para describir el crecimiento animal, el cual no sigue una tendencia lineal, de allí que sea necesario explorar modelos no lineales. Además, las estimaciones de parámetros interpretables biológicamente de una función de crecimiento están asociadas a las características de importancia económica de los animales, y así constituyen una alternativa para los programas de selección (Agudelo-Gómez et al., 2007).

El presente estudio tuvo como objetivo determinar el óptimo técnico y económico para la saca única de reproductores del cuy criollo en la EEA Chumbibamba del Instituto Nacional de Innovación Agraria. Esto permitirá determinar límites de saca óptima de reproductores basado en la ganancia de peso vivo y manteniendo los aspectos de bienestar del cuy.

\section{Materiales y Métodos}

\section{Área de Estudio}

La EEA Chumbibamba del INIA se localiza en el distrito de Talavera, provincia
Andahuaylas, Región Apurímac, ubicado al sureste del Perú (Figura 1), aproximadamente a $2843 \mathrm{msnm}$. La localización ecológica corresponde a bosque sierra subtropical media alta. El clima es variado con frío templado y la temperatura oscila entre 18 y $28^{\circ} \mathrm{C}$.

\section{Crianza de Cuyes en la EEA Chumbi- bamba}

La crianza de cuyes se inició en 2003 con las razas Perú, Inti y Andina. Actualmente cuenta con un galpón exclusivo de cuyes criollos denominado «Banco de Germoplasma del Cuy Nativo»; que fue iniciado mediante la colecta de cuyes nativos (150 reproductores) en las provincias de la región Apurímac (Abancay y Andahuaylas). A junio de 2020, el Banco de Germoplasma alberga 572 ejemplares, que se distribuyen en 65 lactantes, 237 recrías, 168 reproductores y 102 cuyes descarte criados bajo un sistema tecnificado.

\section{Fuente de Datos}

Los datos para el estudio fueron obtenidos de los registros del Banco de Germoplasma de Cuyes Nativos. La información biológica corresponde a los pesos vivos semanales: peso al nacimiento, y pesos a $\operatorname{los} 7,14$, $21,28,35,42,49,56,63,70,77,84,91,98$, 105 y 112 días de edad. La alimentación fue ad libitum y estuvo basada en una dieta compuesta con el $70 \%$ de alfalfa (Medicago sativa) y $30 \%$ de afrecho. Se consideró como variable respuesta la rentabilidad del negocio de cuyes expresada en la tasa de descuento con relación al tiempo de vida útil productiva de los cuyes como reproductores en la EEA Chumbibamba bajo cuatro escenarios de tasa de descuento $(9,12,15$ y $30 \%)$.

\section{Metodología}

Inicialmente se realizó un análisis exploratorio de los parámetros técnicos asociados al comportamiento en el tiempo del 




Figura 1. Ubicación geográfica de la Estación Experimental Agria (EEA) Chumbibamba del Instituto Nacional de Investigación Agraria (INIA), distrito Talavera, provincia Andahuaylas, región Apurímac

peso vivo y la tasa de crecimiento semanal. Luego, se plantearon dos modelos que permiten simplificar el comportamiento de la especie considerando las principales variables.

El planteamiento de un modelo bioeconómico considera el comportamiento biológico de la especie, el cual considera implícitamente la capacidad de carga de un nicho ecológico y/o ecosistema, entonces la población se acercará a este nivel y se mantendrá en sus alrededores con el paso del tiempo y permitirá encontrar soluciones para su manejo sostenible.

El primer modelo comprendió la aplicación de un modelo biológico equivalente a determinar una forma funcional que relaciona el comportamiento del peso vivo con la edad según sexo (Tietenberg y Lewis, 2018;
Quispe y Lavado, 2019). El uso de una forma funcional permite modelizar el comportamiento de las variables en análisis y, a partir de ello, es posible analizar escenarios respecto al crecimiento del recurso (Maldonado 2008). Para señalar el óptimo biológico se realizaron regresiones de diversos modelos polinómicos y de diferente grado. Se seleccionó el modelo de mejor ajuste basado en el coeficiente de determinación $\left(\mathrm{R}^{2}\right)$, se desagregó por sexo y a nivel global. Además, la condición de optimización del modelo biológico se da mediante la expresión (1): $f^{\prime}(t)$ $=f(t) / t$, donde $f(t)$ : Función de producción que relaciona el peso vivo con la edad de la especie, y $t$ : Tiempo. El modelo biológico permitió hallar el valor del óptimo técnico que expresa la máxima edad (en días) para la saca de cuyes hembras y machos como reproductor. Para esto se utilizaron los programas Stata 14 y Derive. 




Figura 2. Comportamiento del peso vivo y tasa de crecimiento del peso vivo en cuyes criollos en la EEA Chumbibamba (Fuente: Registros INIA - EEA Chumbibamba)

El segundo modelo corresponde al modelo bioeconómico de Fisher-Hotelling de rotación única a precio constante denominado «Modelo bioeconómico de saca única de reproductores» (Hotelling, 1991; Tietenberg y Lewis, 2018). Este modelo se aplicó debido al comportamiento estable del precio en vivo del cuy. Se utilizó el programa Derive. El comportamiento del precio cuy fue preestablecido por el INIA, y este se mantuvo constante en $\mathrm{S} / .25$ soles $(1 \mathrm{US} \$=\mathrm{S} / .3 .50$ a agosto de 2020), independientemente del sexo del animal. Este modelo permitió determinar el tiempo de extracción óptimo del recurso dado un nivel de rentabilidad que se expresa en términos porcentuales y se denomina tasa de descuento. En tal sentido, el modelo debe cumplir la condición de optimización que señala la siguiente expresión: $f^{\prime}(t) / f(t)=r$, donde $f(t)$ : Función de producción que relaciona el peso vivo en función a la edad de la especie, $f^{\prime}(t)$ : Primera derivada de la función de producción que relaciona el peso vivo en función a la edad de la especie, y $r$ : Tasa de descuento o costo de oportunidad del recurso. Con base a esta expresión se determinaron los tiempos óptimos económicos para la saca de reproductores.

Dado que la solución del modelo FisherHotelling es única, se realizaron cuatro escenarios según la tasa de descuento ( 9 al 30\%), donde un $9 \%$ es el indicador de rentabilidad en proyectos de inversión pública y $30 \%$ es el promedio del costo de oportunidad del sector turismo. Finalmente, se realizaron las comparaciones de los resultados de ambos modelos, que corresponden a valores óptimos técnico y económico.

\section{Resultados y Discusión}

\section{Parámetros Técnicos}

Los parámetros productivos (peso al nacimiento y destete a los 15 días y ganancia de peso) del cuy nativo o criollo criado en la 
Cuadro 1. Promedio de peso al nacimiento, al destete y ganancia de peso al destete (15 días) del cuy criollo según sexo en la EEA Chumbibamba del Instituto Nacional de Investigación Agraria (INIA), distrito Talavera, provincia Andahuaylas, región Apurímac, Perú

\begin{tabular}{lccccc}
\hline \multirow{2}{*}{ Sexo } & \multicolumn{3}{c}{ Nacimiento } & \multicolumn{3}{c}{ Destete } \\
\cline { 2 - 6 } & $\mathrm{n}$ & $\begin{array}{c}\text { Peso } \\
(\mathrm{g})\end{array}$ & $\mathrm{n}$ & $\begin{array}{c}\text { Peso } \\
(\mathrm{g})\end{array}$ & $\begin{array}{c}\text { Ganancia de } \\
\text { peso }(\mathrm{g})\end{array}$ \\
\hline Hembra & 153 & $127.07^{\mathrm{b}}$ & 151 & $226.98^{\mathrm{b}}$ & $99.91^{\mathrm{b}}$ \\
Macho & 159 & $133.88^{\mathrm{a}}$ & 154 & $240.23^{\mathrm{a}}$ & $106.35^{\mathrm{a}}$ \\
\hline Promedio & 312 & 130.54 & 305 & 233.67 & 102.69 \\
\hline
\end{tabular}

$a, b$ Letras diferentes en la misma columna indican diferencia significativa $(p<0.05)$

Fuente: Registros INIA - EEA Chumbibamba

EEA Chumbibamba se muestran en el Cuadro 1. En todos los casos se encontró diferencias significativas entre $\operatorname{sexos}(\mathrm{p}<0.05)$.

La Figura 2 muestra el comportamiento del peso del cuy criollo con una frecuencia semanal desde el nacimiento hasta la semana 16 . Se observa que las variaciones en la tasa de crecimiento (cambio porcentual del peso vivo de un periodo a otro) semanal del peso vivo son superiores al $30 \%$ en las dos primeras semanas, para luego decrecer a 22 y $17 \%$ en las siguientes dos semanas, para continuar entre 9 y $5 \%$ hasta el final del estudio ( $9 \%$ y $5 \%$ ), comportamiento que configura una evidente tendencia no lineal creciente.

Al comparar los parámetros de crecimiento con los cuyes mejorados se evidencia el bajo rendimiento en características de importancia por sus innatas características de rusticidad, resistencia a enfermedades y su adaptación al medio (Aliaga et al., 2009). En la naturaleza, los animales tienen distintos ritmos del crecimiento; pues no es un proceso uniforme, sino que se presenta una fase de autoaceleración, seguido de una fase plana y finalmente de una fase de desaceleración a medida que el animal alcanza la madurez (Lawrence et al., 2012).

\section{Modelo Biológico}

El Cuadro 2 muestra los modelos biológicos por sexo y a nivel global, que corresponden a una función polinómica de orden 2 y sus parámetros en función al tiempo y el valor del máximo/óptimo técnico. Los resultados indican que el óptimo productivo para cuyes hembras ocurrirá a 414 días, mientras que en los machos sería a 579 días (490 días a nivel global). El rápido deterioro de la vida productiva de las hembras es inherente al desgaste fisiológico asociado a la gestación y la lactancia que cumplen durante su ciclo de vida (Lonergan et al., 2018).

El crecimiento es un proceso fisiológico común a todos los seres vivos que permite el aumento del tamaño y el peso que refleja la utilización de elementos nutritivos del medio exterior (Lonergan et al., 2018). En tal virtud, el modelo que caracteriza el crecimiento de los animales permite al criador poder determinar la conformación final y la calidad de la canal (Buxadé, 1996). El patrón descrito (Figura 2) tiene un comportamiento propiamente cuadrático inherente a los elementos biológicos, y que a la vez, evidencia un crecimiento lento, fundamentalmente originado por las características de su genotipo. 
Cuadro 2. Coeficientes del modelo biológico del peso vivo en función de la edad en cuyes criollos según sexo

\begin{tabular}{llcll}
\hline Sexo & Variable & Coeficiente & Modelo & $\begin{array}{l}\text { Máximo } \\
\text { técnico }\end{array}$ \\
\hline Cuy hembra & $\mathrm{X}$ & 7.055 & & $\mathrm{X}_{1}=-17.81$ \\
& $\mathrm{X}^{2}$ & -0.018 & $-0.0178 \mathrm{X}^{2}+7.0548 \mathrm{X}+131.33$ & $\mathrm{X}_{2}=414.15$ \\
& Constante & 131.330 & & \\
Cuy macho & $\mathrm{X}$ & 7.701 & & $\mathrm{X}_{1}=-16.94$ \\
& $\mathrm{X}^{2}$ & -0.014 & $-0.0137 \mathrm{X}^{2}+7.7008 \mathrm{X}+134.46$ & $\mathrm{X}_{2}=579.05$ \\
& Constante & 134.460 & & \\
\hline Cuy & $\mathrm{X}$ & 7.338 & & $\mathrm{X}_{1}=-17.46$ \\
& $\mathrm{X}^{2}$ & 0.016 & $-0.0155 \mathrm{X}^{2}+7.3375 \mathrm{X}+132.9$ & $\mathrm{X}_{2}=490.85$ \\
\hline
\end{tabular}

Variable independiente $X$ (Edad) expresado en días

Cuadro 3. Valores óptimos económicos de saca única en cuyes criollos según sexo bajo cuatro escenarios de tasa de descuento

\begin{tabular}{cccc}
\hline \multirow{2}{*}{ Escenario } & \multicolumn{3}{c}{ Periodo de saca } \\
\cline { 2 - 4 } & Hembra & Machos & Ambos sexos \\
\hline $\mathrm{r}=9 \%$ & $\mathrm{X}_{1}=-6.99 ; \mathrm{X}_{2}=425.65^{*}$ & $\mathrm{X}_{1}=-6.04 ; \mathrm{X}_{2}=590.4^{*}$ & $\mathrm{X}_{1}=-6.60 ; \mathrm{X}_{2}=502.2^{*}$ \\
$\mathrm{r}=12 \%$ & $\mathrm{X}_{1}=-9.64 ; \mathrm{X}_{2}=422.6^{*}$ & $\mathrm{X}_{1}=-8.73 ; \mathrm{X}_{2}=587.5^{*}$ & $\mathrm{X}_{1}=-9.27 ; \mathrm{X}_{2}=499.3^{*}$ \\
$\mathrm{r}=15 \%$ & $\mathrm{X}_{1}=-11.25 ; \mathrm{X}_{2}=420.9^{*}$ & $\mathrm{X}_{1}=-10.36 ; \mathrm{X}_{2}=585.8^{*}$ & $\mathrm{X}_{1}=-10.89 ; \mathrm{X}_{2}=497.6^{*}$ \\
$\mathrm{r}=30 \%$ & $\mathrm{X}_{1}=-14.51 ; \mathrm{X}_{2}=417.5^{*}$ & $\mathrm{X}_{1}=-13.63 ; \mathrm{X}_{2}=582.4^{*}$ & $\mathrm{X}_{1}=-14.16 ; \mathrm{X}_{2}=494.2^{*}$ \\
\hline
\end{tabular}

Elaboración propia, basado en condición de optimización

$*(p<0.05)$.

Las diferencias en los valores del punto máximo técnico entre machos y hembras se atribuyen a la capacidad genética inherente a los machos de lograr mayores índices de crecimiento prenatal y postnatal, especialmente por factores hormonales que determinan una tasa metabólica más acentuada (Lonergan et al., 2018). En cambio, la vida reproductiva de la hembra se caracteriza por ciclos repeti- dos de gestación y lactancia; este último se sustenta en las reservas corporales de grasas y proteínas, cuya capacidad en el tiempo se agota a medida que ocurren los partos subsiguientes (Lawrence et al., 2012). Biológicamente la esperanza de vida de los cuyes se prolonga hasta los ocho años (Rofes y Wheeler, 2003); empero, zootécnicamente la vida productiva de los cuyes mejorados es solo de un año (Aliaga et al., 2009). 
En conjunto, el modelo biológico permite visualizar que los cuyes hasta que alcancen la edad óptima (plena madurez) sostienen las diferencias en la capacidad de ganancia de peso, consumo de alimento y conversión alimenticia; después de aquel tiempo, dichas aptitudes de producción y rentabilidad resultan ineficientes en perjuicio de la fertilidad y fecundidad de la especie.

\section{Modelo Bioeconómico}

El Cuadro 3 muestra los valores óptimos económico del modelo de saca única de reproductores a precio constante por sexo y a nivel global, y bajo escenarios de rentabilidad. En cuyes hembras, a una tasa de descuento del $9 \%$ el periodo óptimo de saca ocurriría a los 425 días, pero si la tasa de descuento fuese del $30 \%$ el periodo óptimo de saca sería a 417 días. En cuyes machos, con estas tasas de descuento los periodos óptimos de saca serían a 590 y 582 días. En tal perspectiva, a mayores niveles de rentabilidad los tiempos de saca se reducen, lo cual sugiere una mayor presión sobre la crianza. Desde el punto de vista económico, la incorporación de precios y el costo de oportunidad del recurso implican aplicar estrategias de manejo que intensifiquen el uso racional de los cuyes reproductores en la granja a fin de lograr niveles de rentabilidad, pero que no alteren el desarrollo biológico de la especie. Una de las características de la especie es la alta precocidad, por ende, las granjas albergan cantidades considerables de estos animales, y los costos que incurren son significativos dada la cantidad presente. Es por esto la necesidad de considerar tiempos óptimos para la saca y así lograr determinados niveles de rentabilidad.

En la literatura revisada, la variación del tamaño corporal de los animales se describe a través de modelos de ajuste de una función, sean estas lineales, polinómicas, Gompertz o Bertalanffy, por mencionar algunas (Agudelo-Gómez et al., 2007; Noguera et al., 2008). El valor del modelo biológico radica en la capacidad de predecir la vida productiva de los cuyes. Esto significa que racionalmente la máxima producción de los cuyes reproductores debería programarse hasta que alcance su periodo de madurez; es decir, desde que ingresa a la reproducción (3-4 meses) donde se alcanza una condición corporal adecuada para lograr no solo el suficiente número de partos, sino el logro de crías con los pesos vivos adecuados. Posterior a este periodo, los niveles de fertilidad y fecundidad se reducen (Chauca et al., 1997; Aliaga et al., 2009). Lo evidente es que la tasa de crecimiento es el resultado de la combinación de dos componentes: el genético y el ambiental (Chauca, 1994; Huamán et al., 2019).

Esta investigación considera el aspecto productivo y el económico; dado que el cuy no solo es una especie destinada al autoconsumo, sino que se ha tornado transable en los mercados locales y regionales. En la perspectiva económica, la mayor presión en el costo de oportunidad del capital, de 9 a $30 \%$, implica no solo reducir el tiempo de saca de los reproductores, sino que se debe intensificar el manejo productivo y reproductivo de los animales a fin de lograr el mayor volumen de cuyes para la venta o reposición (Aliaga et al., 2009).

Al combinar el modelo biológico con el modelo bioeconómico se busca garantizar el desarrollo corporal bajo un escenario de rentabilidad, dado que el modelo permite encontrar el tiempo en el cual la especie logre su desarrollo corporal que se expresa en el desarrollo del lomo y los cuartos traseros, a la vez incrementan la profundidad y el grosor del cuerpo. Paralelamente, el crecimiento muscular se ralentiza y el mayor aumento de peso se debe a la grasa; sin embargo, la deposición de grasa origina menores tasas de ganancia de peso y de crecimiento (Lonergan et al., 2018). Mientras que, el modelo bioeconómico permite asociar al tiempo del modelo biológico un determinado nivel de rentabilidad que beneficie al productor. 


\section{ConClusiones}

- Los parámetros productivos de pesos al nacer $(127.0$ y $133.8 \mathrm{~g})$, al destete $(227.0$ y $280.2 \mathrm{~g}$ ) y las ganancias de peso vivo (99.9 y $106.4 \mathrm{~g})$ entre el nacimiento y el destete de cuyes nativos fueron estadísticamente diferentes entre sexos $(p<0.05)$. La ganancia de peso vivo fue creciente en las dos primeras semanas, para luego ser decrecientes y a partir de la quinta semana se tornan constantes hasta alcanzar la madurez.

- Los modelos biológicos y sus parámetros en función al tiempo y el valor del máximo técnico permiten predecir la vida productiva óptima de los cuyes reproductores (Hembras: 414 días; machos: 579 días).

- Los valores óptimos del modelo bioeconómico de saca única a precio constante en cuyes hembras y machos fue de 425 y 590 días, respectivamente.

\section{Literatura Citada}

1. Agudelo-Gómez D, Cerón-Muñoz M, Restrepo L. 2007. Modelación de funciones de crecimiento aplicadas a la producción animal. Rev Colomb Ciencias Pecu 20: 157-173.

2. Aliaga L, Moncayo R, Rico $E$, Caycedo A. 2009. Producción de cuyes. Lima: Univ. Católica Sedes Sapientiae. $808 \mathrm{p}$.

3. Buxadé C. 1996. Zootecnia. Bases de producción animal. Producciones cuniìcula y aviìcolas alternativas. Madrid, España: Mundi-Prensa. 350 p.

4. Chauca L. 1994. Investigaciones en cuyes. Lima: INIA. [Internet]. Disponible en: https://idl-bnc-idrc.dspacedirect.org/bitstream/handle/10625/16040/ 100159.pdf?sequence $=1$
5. Chauca L. 2007. Logros obtenidos en la mejora genética del cuy (Cavia porcellus). Experiencias del INIA. En: XX Reunión de la Asociación Latinoamericana de Producción Animal. Cusco, Perú.

6. Chauca L. 2020. Manual de crianza de cuyes. Lima: INIA. $53 \mathrm{p}$

7. Chauca L, Muscari J, Higaona R. 1997. Proyecto sistemas de producción de crianzas familiares Perú 93-0028. Tomo II. Lima: Centro de Estudios y Desarrollo Agrario del Perú. $113 \mathrm{p}$.

8. [FAO] Organización de las Naciones Unidas para la Agricultura y la Alimentación. 2012. Ganadería mundial 2011. La ganadería en la seguridad alimentaria. McLeod A (ed). Roma: FAO. [Internet]. Disponible en: http:// www.fao.org/3/i2373s/i2373s00.pdf

9. Huamán M, Killerby M, Chauca L. 2019. Manual de bioseguridad y sanidad en cuyes. Lima: Instituto Nacional de Investigación Agraria. $86 \mathrm{p}$.

10. [INEI] Instituto Nacional de Estadística e Informática. 2012. IV Censo Nacional Agropecuario. [Internet]. Disponible en: http://censos.inei.gob.pe/ Cenagro/redatam/

11. [INIA] Instituto Nacional de Innovación Agraria. 2018. Rol del INIA en el proceso histórico de la investigación agraria en el Perú. Lima: INIA. 191 p.

12. Hotelling H. 1991. The economics of exhaustible resources. Bull Math Biol 53:281-312. doi: 10.1007/BF02464433

13. Jorgensen S, Bendoricchio G. 2001. Fundamentals of ecological modelling. $3^{\text {rd }}$ ed. Amsterdam: Elsevier Science. 544 p.

14. Lawrence T, Fowler V, Novakofski J. 2012. Growth of farm animals. $3^{\text {rd }}$ ed. CABI. 368 p.

15. Lonergan SM, Topel DG, Marple DN. 2018. The science of animal growth and meat technology. USA: Academic Press. 280 p. 
16. Maldonado J. 2008. Economía de recursos naturales: aplicaciones de la economía computacional a la solución de problemas dinámicos. Bogotá: Universidad de los Andes. 229 p.

17. Noguera RR, Pereira RL, Solarte CE. 2008. Comparación de modelos no lineales para describir curvas de crecimiento en cuyes (Cavia porcellus) desde el nacimiento hasta la edad de sacrificio. Livest Res Rural Dev 20(5). [Internet]. Disponible en: http:// www.lrrd.org/lrrd20/5/nogu20079.htm

18. Pomboza-Tamaquiza P, Velastegui G, Damián-Barajas A. 2016. Roles de la crianza de cuyes (Cavia porcellus) en sistemas agroecológicos y en la sociedad rural. Livest Res Rural Dev 28(6).
[Internet]. Disponible en: https://lrrd.cipav.org.co/lrrd28/6/pomb-28110.html

19. Quispe DM, Lavado K. 2019. Modelo bioeconómico de esquila única en el manejo de la fibra de vicuña. Arch Zootec. 68: 348-355. doi:10.21071/ az.v68i263.4192

20. Rofes J, Wheeler JC. 2003. Sacrificio de cuyes en los Andes: El caso de El Yaral y una revisión biológica, arqueológica y etnográfica de la especie Cavia porcellus. Archaeofauna 12: 29-45.

21. Sandoval CA, Sarmiento L, Santos RH. 2013. ¿Qué son y cuál es el papel de las especies menores? Bioagrociencias 6: 20-27.

22. Tietenberg T, Lewis L. 2018. Environmental and natural resource economics. $11^{\text {th }}$ ed. New York: Routledge. 586 p. 